\title{
An empirical analysis on the arterial fundamental diagram
}

\author{
Xinkai $\mathrm{Wu}^{\mathrm{a}}$, Henry X. Liu ${ }^{\mathrm{a}, *}$, Nikolas Geroliminis ${ }^{\mathrm{b}}$ \\ ${ }^{a}$ Department of Civil Engineering, University of Minnesota, Twin Cities, United States \\ ${ }^{\mathrm{b}}$ School of Architecture, Civil and Environmental Engineering, École Polytechnique Fédérale de Lausanne, Switzerland
}

\section{A R T I C L E I N F O}

\section{Article history:}

Received 7 January 2009

Received in revised form 1 June 2010

Accepted 3 June 2010

\section{Keywords:}

Arterial fundamental diagram (AFD)

Traffic signal

$g / C$ ratio

Queue-Over-Detector (QOD)

Signal coordination

\begin{abstract}
A B S T R A C T
For uninterrupted traffic flow, it is well-known that the fundamental diagram (FD) describes the relationship between traffic flow and density under steady state. For interrupted traffic flow on a signalized road, it has been recognized that the arterial fundamental diagram (AFD) is significantly affected by signal operations. But little research up to date has discussed in detail how signal operations impact the AFD. In this paper, based upon empirical observations from high-resolution event-based traffic signal data collected from a major arterial in the Twin Cities area, we study the impacts of $g / C$ ratio, signal coordination, and turning movements on the cycle-based AFD, which describes the relationship between traffic flow and occupancy in a signal cycle. By microscopically investigating individual vehicle trajectories from event-based data, we demonstrate that not only $g / C$ ratio constrains the capacity of a signalized approach, poor signal coordination and turning movements from upstream intersections also have significant impact on the capacity. We show that an arterial link may not be congested even with high occupancy values. Such high values could result from queue build-up during red light that occupies the detector, i.e. the Queue-Over-Detector (QOD) phenomenon discussed in this paper. More importantly, by removing the impact of QOD, a stable form of AFD is revealed, and one can use that to identify three different regimes including under-saturation, saturation, and over-saturation with queue spillovers. We believe the stable form of AFD is of great importance for traffic signal control because of its ability to identify traffic states on a signal link. (c) 2010 Elsevier Ltd. All rights reserved.
\end{abstract}

\section{Introduction}

The fundamental diagram (flow-density or flow-occupancy relationship, FD) is of great importance in advancing traffic flow theory and analyzing traffic behavior. Over the years such relationship has been explored by many researchers, but majority of previous investigations focus on uninterrupted traffic flow on freeway. Relevant research for signalized arterials is limited. It has been recognized that the arterial fundamental diagram (AFD) is significantly affected by signal operations, but no literature up to date has discussed the inner mechanism of such impact. One reason behind this is the lack of highresolution traffic data on signalized arterials in the past. As high-resolution traffic data from signalized arterials become accessible nowadays, this paper aims to fill in this gap.

In this paper, our goal is to explore the impacts of signal operations on the AFD by analyzing the cycle-based flow-occupancy data from a "point" detector on a signal link. With the high-resolution "event-based" traffic signal data (both vehicledetector actuations and signal phase changes are considered as "events"), we are able to reconstruct the trajectories of each vehicle passing the detector. By microscopically investigating these individual vehicle trajectories, we describe that how the AFD is impacted by the $g / C$ ratio, signal coordination, and turning movements. Additionally, we show that the simple plot of

\footnotetext{
* Corresponding author. Address: 500 Pillsbury Dr. S.E., Minneapolis, MN 55455, United States. Tel.: +1 6126256347 ; fax: +1 612 626 7750.

E-mail address: henryliu@umn.edu (H.X. Liu).
} 
the flow-occupancy diagram from detector data cannot be utilized directly to analyze traffic flow characteristics on an arterial link, due to the Queue-Over-Detector (QOD) phenomenon, as we will explain later. More importantly, by removing the impact of QOD, a stable form of AFD is revealed, and one can use that to identify three different regimes including undersaturation, saturation, and over-saturation with queue spillovers. We believe the stable form of AFD is of great importance for traffic signal control because of its ability to identify traffic states on a signal link.

Our argument in this paper is not a theoretical one; instead, it is solely empirical, based on the analysis of the field data collected from a major arterial in the Twin Cities area. It is not our intent to downgrade the importance of any theoretical rationale. Rather, we believe that as more detailed data on arterial roads are now available, it is more desirable to understand empirical data from real-world before building an analytical model.

This paper is organized as follows. Section 2 discusses some relevant work to set the background for the current analysis. Detailed description of field data and empirical observations of flow-occupancy diagrams are included in Section 3, followed by the explanations of the impact of signal operations on the AFD in Section 4. In Section 5 we discuss the inner mechanism of the QOD phenomenon. We further reveal a stable form of flow-occupancy diagram after removing QOD in Section 6. Conclusions are offered in the final section.

\section{Background}

Lighthill and Whitham in their classical work (Lighthill and Whitham, 1955) first described the FD as that "... at any point of the road the flow (vehicles per hour) is a function of the concentration (vehicles per mile)..." Over the past decades, traffic scientists developed a wide range of mathematical models for uninterrupted traffic flow. The seminal work was done by Greenshields (1935), who firstly observed a flow-density diagram and derived a parabolic equation for the flow-density curve based on a linear speed-density relationship. Following Greenshields, different relationships of speed and density were proposed based on empirical data for freeways, such as Greenberg's logarithmic relationship (1959) and Underwood's transposed exponential function (1961). An interesting phenomenon, as pointed out by Edie (1961), is that traffic behavior appears to be different at high and low concentrations. A discontinuous exponential form, which combines the Greenberg and Underwood curves, therefore was proposed by Edie (1961). Later, Koshi et al. (1983) gave an empirically-based discussion of the flow-density relationship and asserted that a discontinuity between uncongested and congested flow regions "becomes obvious some distance upstream of the bottleneck". Using empirical freeway data, Hall et al. (1986) further provided several alternative flow-occupancy relationships to describe the discontinuity of the FD.

Most mathematical models developed above assume that traffic is in steady state, which is also called "spatially homogeneous" or "equilibrium" conditions. It should be noted, however, that real traffic patterns, especially under congested conditions, are spatially non-homogeneous. This has been demonstrated empirically (Hall et al., 1986) that at low densities a clean linear curve can be observed, while strong fluctuations exist "which prevents a direct evaluation of the functional form at high densities." (Chowdhury et al., 2000) To accommodate this, Kerner's model (2004) assumes a range of flow rates for a given value of density under congested conditions.

To date, most research on the FD focuses on uninterrupted traffic flow. Related research on interrupted traffic flow, particularly at signalized arterials, is limited. This is partly due to the difficulty of arterial data collection, as most of the traffic signal systems do not record and archive traffic event data; and partly because of the complexity of the interrupted nature of arterial flow, as arterial flow, "by its very nature, displays frequent uneven fluctuations" (Hallenbeck et al., 2008).

For an AFD, it is known that the capacity of a signalized link is constricted by the "green/cycle ratio". But whether coordination or offset has any effect on the AFD is still an open question. Gartner and Wagner (2004) conducted a simulation study, in which they examined the relationship between flow and density on rings. They simulated the ring for a limited range of signal timings and unveiled several regularities. These regularities, however, cannot be extrapolated to form a general theory because simulation only speaks to the range of simulated parameters. An example of a regularity that cannot be extrapolated is the independence between system capacity and signal offsets because, as we will show with empirical data in this paper, offsets do affect the capacity when turning volumes from upstream minor approaches remain constant.

Daganzo, together with Geroliminis, provided empirical evidences for the existence of an urban-scale macroscopic FD; and later developed an analytical model to estimate the macroscopic FD based on variational theory, for a network with many intersections (Daganzo and Geroliminis, 2008; Geroliminis and Daganzo, 2008). Helbing (2009) derived an analytical model for the urban FD by applying a utilization-based approach and his research focuses more on the relationship between average travel time and utilization (or the number of delayed vehicles), instead of flow vs. density relation. Both of the above studies do not provide an explanation for the scatter in the FD for an arterial link, and their analysis applies when the number of links is large.

It is apparent that empirical studies of the AFD are limited. This research aims to fill this gap by analyzing the cycle-based flow-occupancy diagrams on the basis of the high-resolution event-based traffic signal data collected from one major arterial in the Twin Cities area. We focus on the cycle-based AFD, not only because signal operations interrupt traffic flow in a cyclic manner, leading to non-steady traffic states within a cycle; but more importantly such diagram indicates traffic states (under-saturation, saturation, and over-saturation) on an arterial link for each cycle and can be used to describe how traffic states transit from cycle to cycle. Although the FD is originally defined as the flow-density relationship, we do not attempt to convert raw occupancy data to more commonly used density parameter. The reason is that "converting measured occupancy to calculated density inherently creates an unnecessary degree of uncertainty." (Hall et al., 1986) Therefore, the flow-occupancy diagram, instead of flow-density diagram, is the subject investigated in the research. 


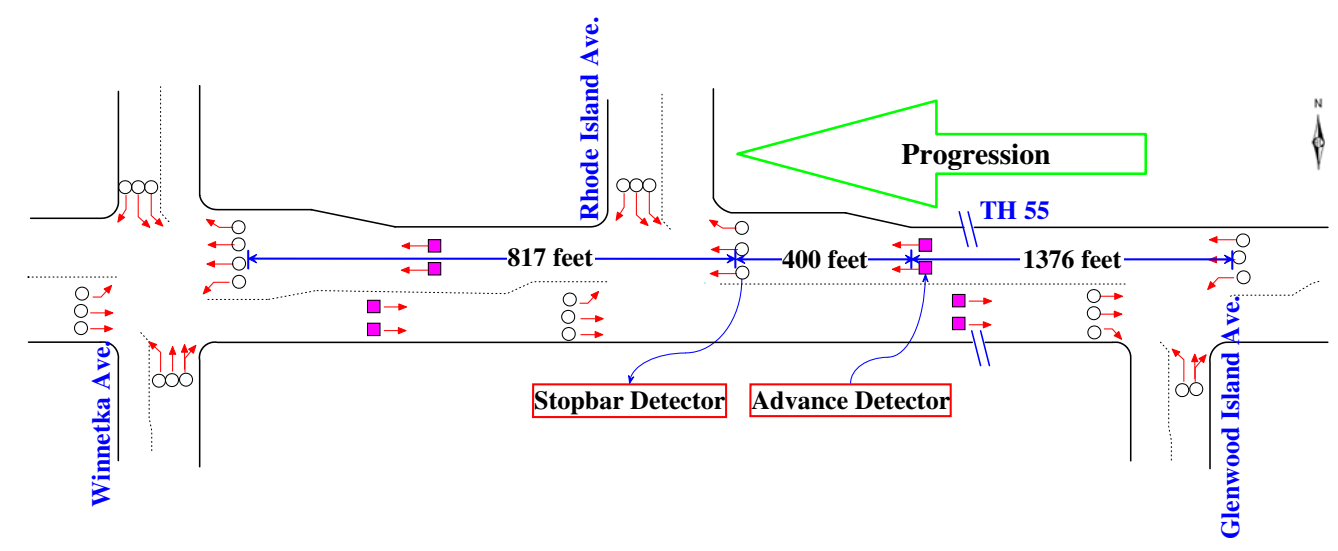

Fig. 1. Data collection site on TH55 in Minneapolis, Minnesota.

\section{Field data collection and observations}

\subsection{Field data collection}

The data utilized in this research is collected by the SMART-SIGNAL (Systematic Monitoring of Arterial Road Traffic Signals) system (Liu and Ma, 2009; Liu et al., 2009). The SMART-SIGNAL system can continuously collect and archive high-resolution event-based traffic signal data. Event data provide start and end times of each vehicle-detector actuation and signal phase change, therefore traffic flow variables (flow and occupancy) and signal phase information can be extracted from the raw data. We should note that detector occupancy herein is referred to the percentage of time that a detector is being occupied. The SMART-SIGNAL system has been installed on six consecutive intersections on Trunk Highway 55 (TH55) in the Twin Cities area since February 2008. Fig. 1 shows the sketch layout of the data collection site on TH55. All intersections shown in the figure are equipped with vehicle-actuated signals, with advance detectors typically located $400 \mathrm{ft}$. upstream from the stop line for green extension on the major approach, and stop-bar detectors located right behind the stop line for presence detection.

Signal information including green, red, amber, and cycle length is obtained from signal events. During morning and evening peak hours, the cycle length of all intersections is constant (180 s) with a few exceptions (due to preemptions) but green time varies with $\mathrm{g} / \mathrm{C}$ ratio ranging from 0.65 to 0.75 for the major approach of TH55 and $0.25-0.35$ for the minor approaches. In this research, we aggregate flow and occupancy data collected from one advance detector and one stop-bar detector on a major approach for each cycle, and then analyze the cycle-based flow-occupancy diagram.

\subsection{Observations}

Cycle-based flow-occupancy diagrams extracted from the stop-bar and advance detectors at the intersection of Rhode Island Ave (see Fig. 1) are presented here. Analysis on other intersections at the study site shows similar results. Fig. 2a and b demonstrate the flow-occupancy plots for 1 day. The AM data (7:00 am-9:00 am) and PM data (3:30 pm-6:30 pm) are colored by purple ${ }^{1}$ and yellow respectively for comparison.

Compared with the flow-occupancy diagram from stop-bar detector (Fig. 2a), which is greatly scattered due to the frequent stop-and-go traffic near stop-bar detectors, the flow-occupancy diagram from the advance detector (Fig. $2 \mathrm{~b}$ ) has much higher orderliness. It is clear in Fig. $2 b$ that the left hand side (occupancy $\leqslant 0.12$ ) corresponds to an uncongested regime indicated by a roughly straight line, and the right hand side represents a congested regime with high occupancy values. The wide range of congested regime is consistent with the conventional understanding that suggests a trapezoidal form of AFD as green ratio constrains the capacity. However, Fig. 2b indicates that AM (purple) and PM (yellow) peaks have different capacity values. To show that such "two capacity values" phenomenon is not by coincidence, we present the flow-occupancy plots using 2 weeks of data from the advance loop detector in Fig. 3.

"Two capacity values" is an interesting phenomenon observed at signalized arterials. As we will show in the following sections, this phenomenon is closely related to several factors that affect signal operations, including $g / C$ ratio, signal coordination, and turning volumes from the upstream intersections. This research will explain this phenomenon by answering the following two questions:

\footnotetext{
${ }^{1}$ For interpretation of color in Figs. 2, 5, 6, 7, 10, 12 the reader is referred to the web version of this article.
} 

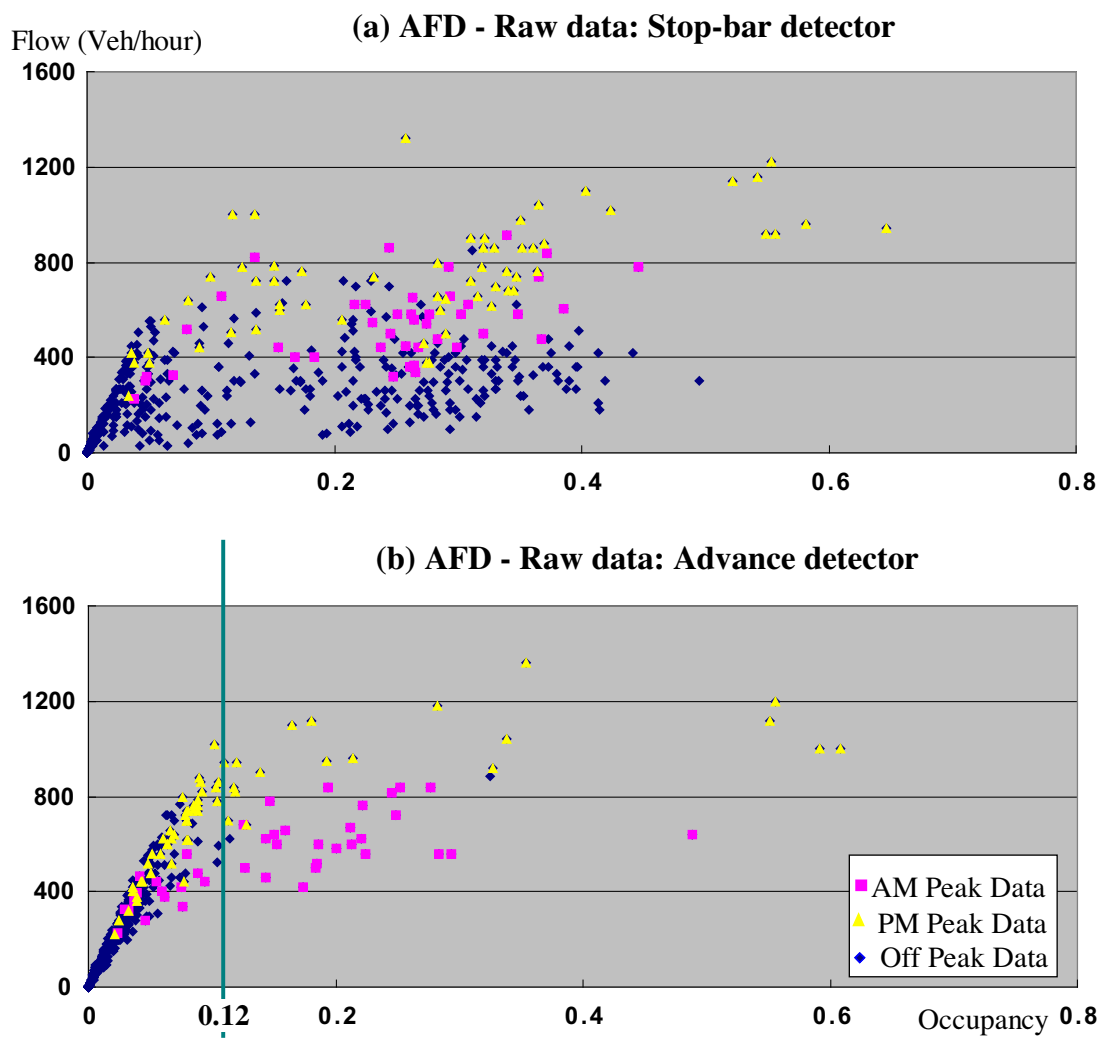

Fig. 2. Cycle-based AFDs using raw detector data: (a) stop-bar detector and (b) advance detector.

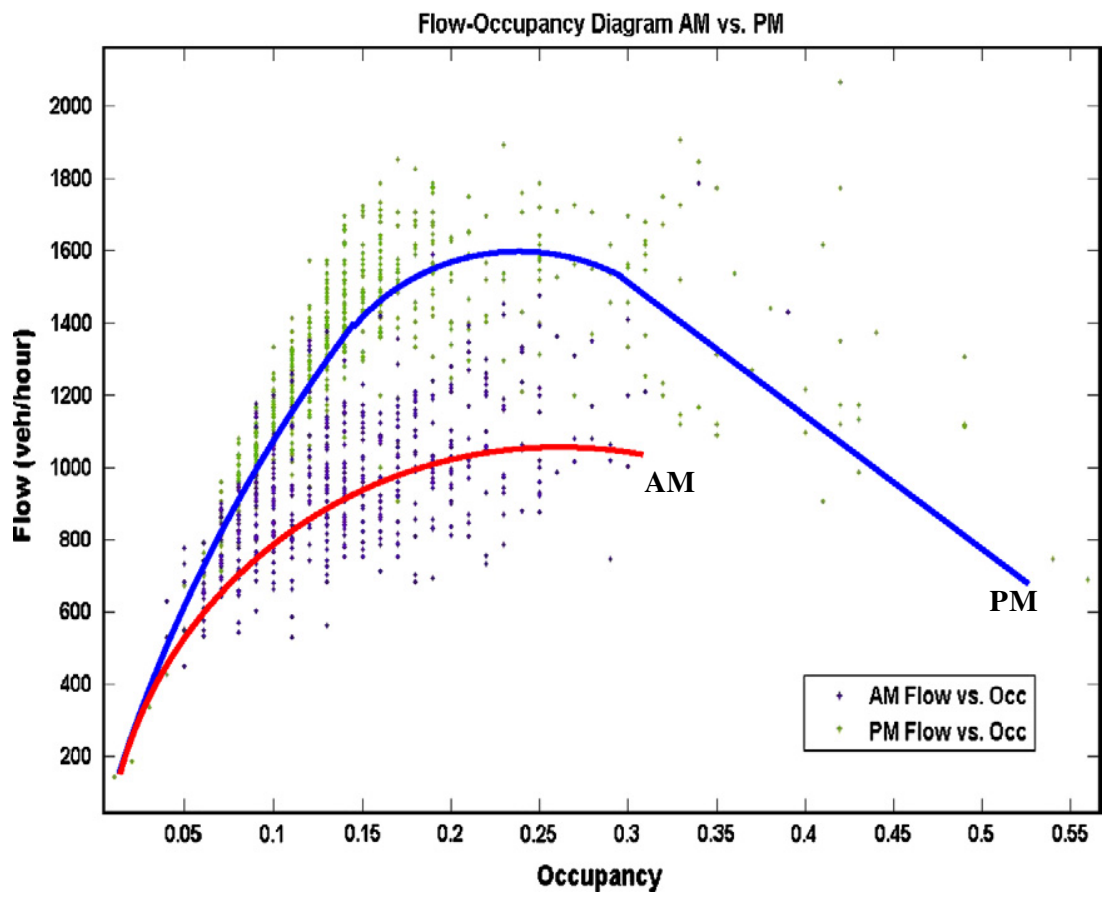

Fig. 3. Cycle-based AFDs for AM and PM peak hours (2 weeks' data). 
(1) Why do different capacity values appear in the flow-occupancy diagrams for the morning and afternoon peaks? It is known that different $g / C$ ratios will lead to different capacity values, but is this the main reason?

(2) Do the scattered points with high occupancy values really indicate congestion on a signal link? For freeway flow-occupancy diagrams, it is widely accepted that larger occupancy usually means higher degree of congestion. Is this true for the arterial flow-occupancy diagram?

\section{The impacts of signal operations on the AFD}

\subsection{The green ratio ( $\mathrm{g} / \mathrm{C}$ ratio)}

An intuitive explanation for the "two capacity values" phenomenon is that AM and PM have different $g / C$ ratios, because the data was collected from an intersection with fully-actuated signal control. $g / C$ ratio varies from cycle to cycle, with an average about 0.65 in the AM and 0.7 in the PM. To see if the impact of different $g / C$ ratios, for the flow-occupancy diagram plotted based on the data collected by the advance detector, the flow rates for the data points in the saturated regime are divided by the corresponding $\mathrm{g} / \mathrm{C}$ ratios. The result is shown in Fig. 4 . Clearly, there are still two distinguishable capacity values for AM and PM, although the difference between the two capacity values are slightly reduced comparing with Fig. 2b. This experiment indicates that $g / C$ ratio is not the main factor that contributes to the "two capacity values" phenomenon for this case.

\subsection{Signal coordination and turning movements}

At arterial/network level, conceivably the capacity may also be impacted by signal coordination because poor coordination may create higher percentage of unutilized green times. Since $g / C$ ratio is not the main factor that contributes to the "two capacity values" phenomenon, it is necessary to investigate the impact of signal coordination and turning volumes from the upstream intersections on the AFD. Fig. 5 visualizes the coordination of three intersections on west-bound TH55 (starting from Douglas Dr., passing through Glenwood Ave. and arriving at Rhode Island Ave). Two continuing cycles in AM and PM peaks are selected for comparison. We should note that eastbound direction (going into Minneapolis) is coordinated for AM peaks and westbound (leaving Minneapolis) for PM peaks. Each point in the figure represents a vehicle event recorded by the advance detectors; and green and red bars represent signal phases. From the figure, we can observe that:

(1) The bandwidth in the AM peak is shorter than that in the PM (around $70 \mathrm{~s}$ vs. $90 \mathrm{~s}$ ). A higher percentage of vehicles are forced to meet a red light in the AM; while in the PM, most vehicles pass through three intersections without stops.

(2) The turning volumes in the AM are lower than those in the PM. During the PM peak, a greater portion of green time outside the bandwidth in Rhode Island intersection is filled by turning movements from Glenwood Ave. than the AM peak.

Now the "two capacity values" phenomenon can be explained. Since coordination bandwidth restricts the input flow from upstream intersections entering downstream links, for a coordinated intersection, the approach capacity that can be achieved is not only constrained by green ratio, but also by bandwidth. A small bandwidth will create unutilized green times, which essentially lead to reduced $g / C$ ratios. Besides coordination, the capacity utilization is also related with turning movements, since unutilized green times created by poor coordination may be filled by the flow coming from the minor approaches at upstream intersections. As indicated in the figure, for the AM peak, the capacity is rarely achieved with the

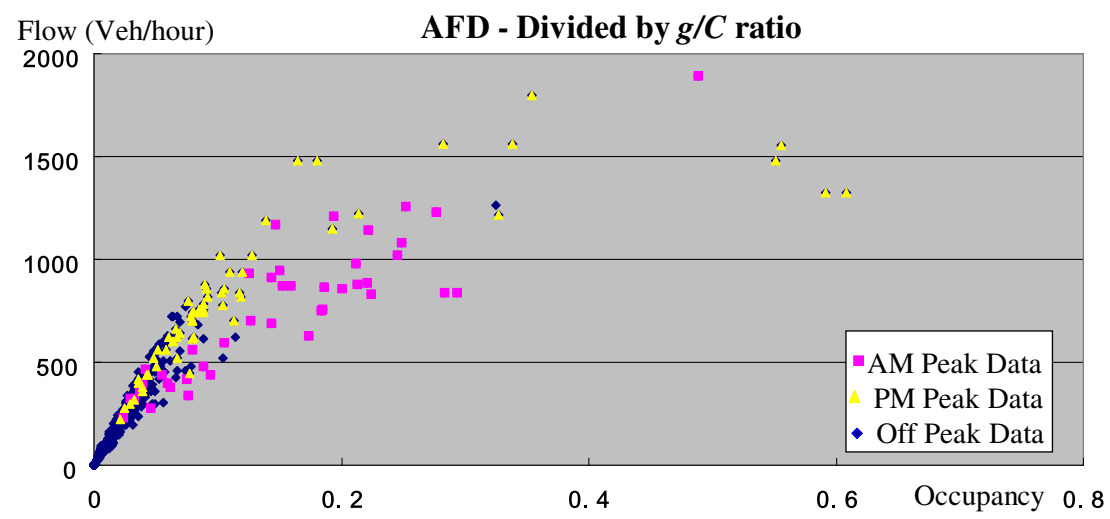

Fig. 4. AFD with flow rates divided by $g / C$ ratios. 
Distance (feet) (a) Coordination \& turning for the AM peak

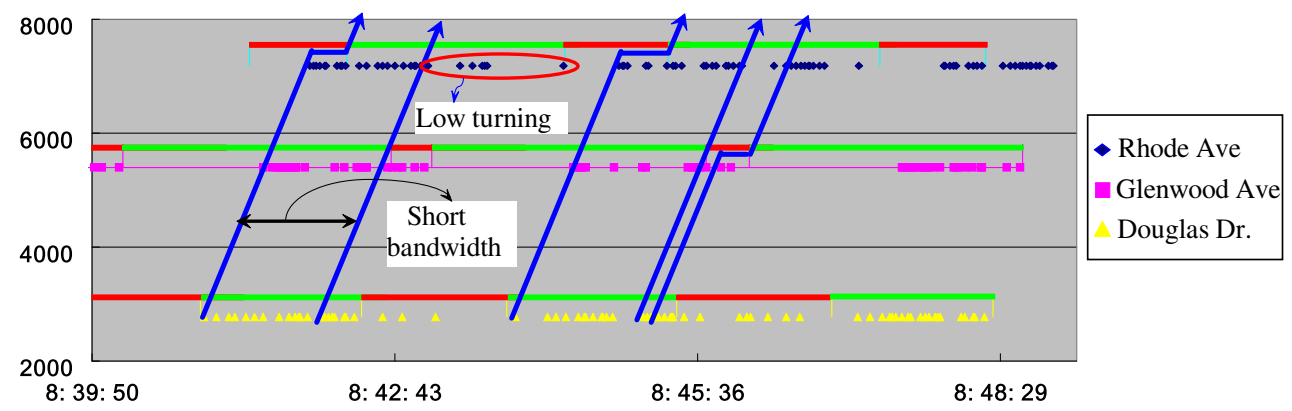

(b) Coordination \& turning for the PM peak

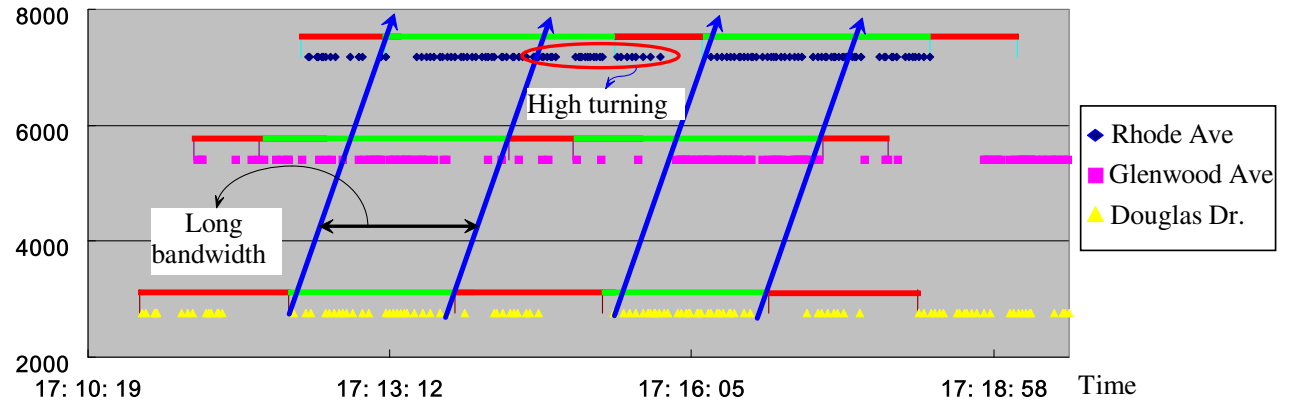

Fig. 5. Coordination and turning movements: (a) AM peak and (b) PM peak.

compounding effects of poor coordination and low turning volumes. In other words, comparing with the PM peak, higher percentage of green time is underutilized in the AM.

Interestingly, although the capacity is rarely achieved in the AM, high occupancies, which usually indicate saturation or over-saturation, occur in a significant number of cycles. Higher occupancy seems contradictory with capacity underutilization. To answer this question, we will introduce the Queue-Over-Detector (QOD) concept in the following section.

\section{Queue-Over-Detector (QOD)}

For a signalized link, when vehicular queue spills back to the location of a detector, a vehicle may stop on the detector for a period of time. This relatively prolonged time period can change detector occupancy significantly. We call this phenomenon as "Queue-Over-Detector" (QOD). QOD only affects the occupancy value while keeping flow rate unchanged. Therefore, with the occurrence of QOD, some data points with low flow rate (theoretically they should stayed in the under-saturated regime of the AFD), will be shifted to the saturated and over-saturated areas.

Generally, there are two types of QOD: the first is caused by the queue formed during red phase (Type I); the second is because of the queue spilling back from downstream intersections, i.e. spill-over (Type II). Type II QOD represents that arterial link is truly congested, while Type I only reflects the presence of transient queues. As Type I QOD is simply because of red signal phase, it is unique to arterial. In this paper, QOD is referred to the Type I, unless otherwise noted.

To verify the impact of QOD, aggregated macroscopic traffic flow data is not enough. More detailed microscopic vehicle trajectories are required. Since event-based data provide both start and end times of each vehicle actuation, such information, together with time-stamped signal phase changes, can be utilized to estimate vehicle trajectories using a simplified carfollowing model, which is similar with the one proposed by Newell (2001). In the simplified model, lane-changing behaviors are not taken into account because we only estimate the trajectories for a relatively short distance (for example, from the location of advance detector to stop line). Vehicles are assumed to accelerate if their speeds are less than free-flow speed ( $55 \mathrm{mph}$, in this study), and decelerate if higher than free-flow speed. The distance between any two vehicles should satisfy safety distance constraint, which is determined by current speeds of two consecutive vehicles, reaction time (about $1.0 \mathrm{~s}$ ), and stop distance (assumed $25 \mathrm{ft}$.). Each vehicle also needs to decide whether passing intersection or not during yellow time based on its current state including its speed, yellow time left, distance with the front vehicle, etc. For each detector actuation event, one vehicle trajectory will be derived.

We now compare two data points in the flow-occupancy diagram with the same flow (600 veh/h) but different occupancy values $(0.213$ vs. 0.064$)$. Note that the flow-occupancy data pair $(600,0.213)$ is from the AM and $(600,0.064)$ from the PM. Vehicle trajectories during these two cycles are derived and presented in Fig. 6. As shown in Fig. 6a, within the first 
selected cycle, more vehicles arrive during the red phase creating a long queue; and one vehicle stops at the advance detector generating an occupancy value of $100 \%$ until the discharge shockwave propagates back to detector. On the contrary, during the second selected cycle (Fig. 6b), only a small portion of vehicles are arriving during red and queue is not long enough to occupy the advance detector, thereby creating $0 \%$ occupancy until the next vehicle arrives after signal turns to green. Such difference in occupancy shifts some data points with relatively small flows from the low occupancy area in the AFD to the high occupancy regime, therefore expands the occupancy and creates occupancy fluctuations.

As discussed above, QOD is the main reason for the occupancy expansion in the AFD. It also implies that higher occupancy does not necessarily indicate congestion, but instead reflects the presence of transient queues. This finding is further verified by a simple experiment that removes QOD impact. In this experiment, we simply move the vehicle stopped on detector a little bit forward or backward for those cycles with long queue (i.e. queue length is longer than the distance from stop line to advance detector). The overall flow and queue length are not changed, but since detector is not occupied by any vehicle now, occupancy suddenly changes from $100 \%$ to a smaller value near $0 \%$ for a period of time, so the QOD impact is removed.

We need to emphasize here that this experiment only removes the occupancy expansion caused by the Type I QOD. If QOD is caused by spill-over, it should not be removed. In detail, the occupancy time created by the queues because of red signal is replaced by a normal occupancy time equivalent to the time required by a free-flowing vehicle that passes detector. The removed time period starts when vehicle arrives at detector and ends when discharge shockwave propagates to the detector (i.e. the time interval between point B and C in Fig. 7). Note the maximum time interval which can be removed is the length between two shockwaves (i.e. between point $A$ and $C$ in Fig. 7, which is equivalent to one red interval). If vehicles arrive before time $A$ or stay on the detector after time $C$, spill-over from the downstream intersection happens. In such situation, only the portion within $\mathrm{A}$ and $\mathrm{C}$ can be replaced by a regular passing time.

Using high-resolution event-based data, Eq. (1) can be used to determine the occupancy expansion for each cycle. Essentially, the expanded occupancy time is the interval between time $T_{B}$ and $T_{C}$ in Fig. 7.

$$
O c c_{i, n}^{\exp }=\frac{T_{C}-\max \left(T_{A}, T_{B}\right)}{C_{i, n}}
$$

where $O c c_{i, n}^{\text {exp }}$ is the occupancy expansion within the $i$ th cycle at the $n$th intersection; $C_{i, n}$ is the cycle length for the $i$ th cycle at the $n^{\text {th }}$ intersection; and $T_{A}, T_{B}$, and $T_{C}$ are time instants presented in Fig. 7.

(a) AM vehicle trajectories - Flow - Occupancy data pair: $(600,0.213)$

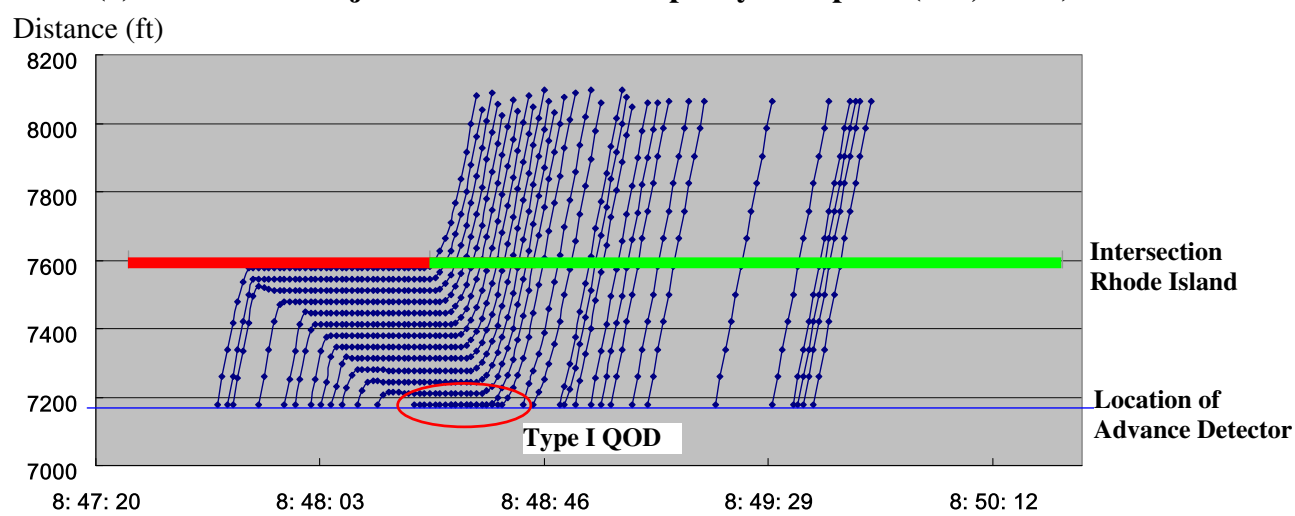

(b) PM vehicle trajectories - Flow - Occupancy data pair: $(600,0.064)$

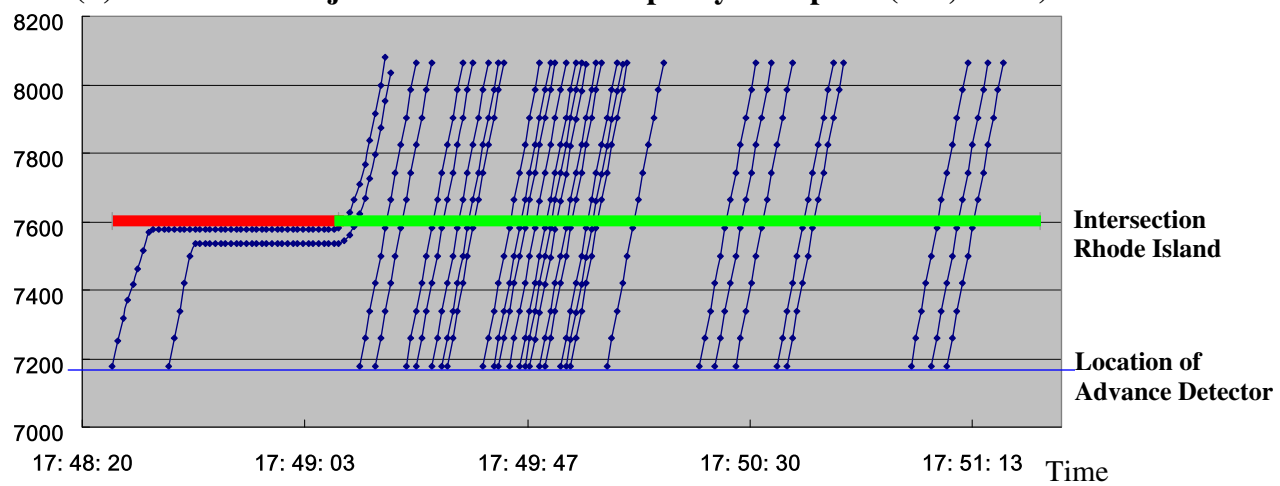

Fig. 6. Vehicle trajectories for two cycles: (a) flow-occupancy $(600,0.213)$ in the AM and (b) flow-occupancy (600, 0.064) in the PM. 


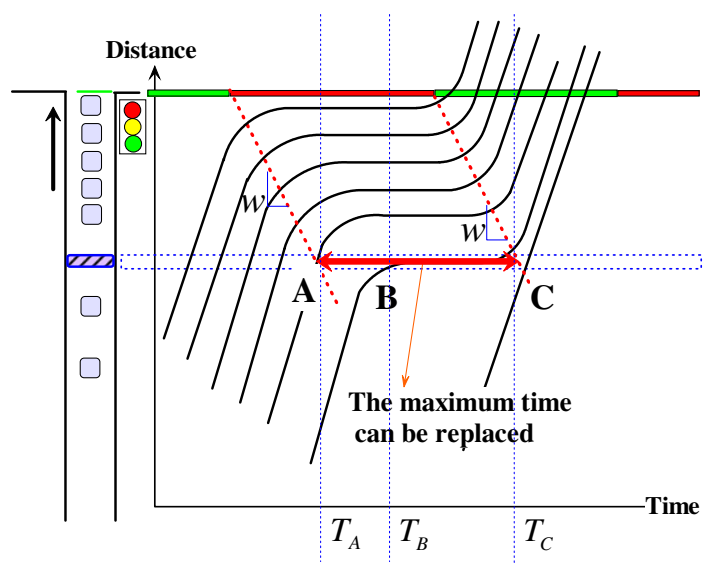

Fig. 7. The maximum QOD time can be replaced.

Assuming the velocity of discharge shockwave is a known constant, then:

$$
O c c_{i, n}^{\exp }=\frac{\left(G_{i, n}+\frac{l_{n}}{w}\right)-\max \left(\left(R_{i, n}+\frac{l_{n}}{w}\right), T_{B}\right)}{C_{i, n}},
$$

where $G_{i, n}$ and $R_{i, n}$ are the time instants when green and red phases start for the $i$ th cycle at the $n$th intersection; $l_{n}$ is the distance between the stop-bar and the location of the advance detector; and $w$ is the velocity of discharge shockwave (see Fig. 7).

We now apply the QOD removal procedure to the raw flow-occupancy diagram. Both revised diagrams for the advance and stop-bar detectors are presented in Fig. 8. Compared with the diagrams drawn by raw data (Fig. 2), the AFDs after

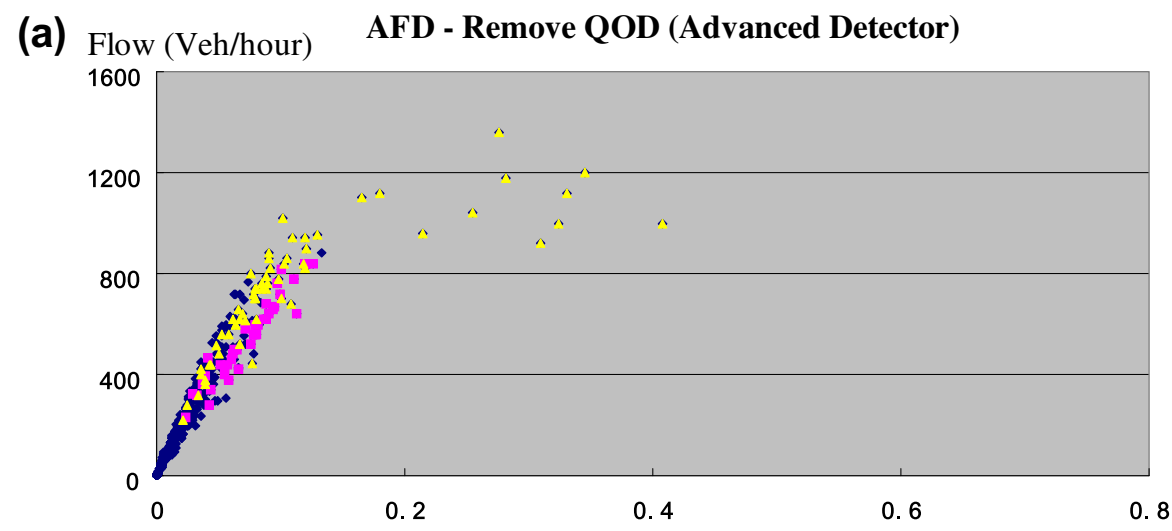

(b)

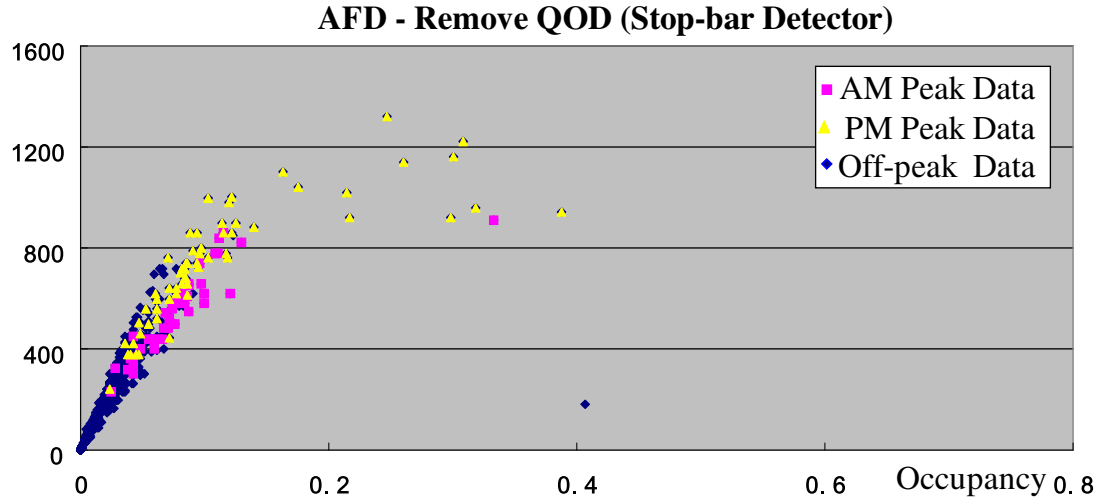

Fig. 8. New AFDs after removing QOD: (a) advance detector and (b) stop-bar detector. 
removing QOD are significantly changed. In particular, the highly scattered cloud in the flow-occupancy diagram for the stop-bar detector disappears, and the two revised flow-occupancy diagrams now have a similar shape.

Carefully comparing the data points before and after removing QOD, Fig. 9 presents the shifting of each data point collected by the advance detector for AM and PM peak hours respectively. Interestingly, for AM peak, almost all the points which originally stay at the right hand side shift back to the left hand side; and the revised diagram has a nice shape (almost linear). This observation also provides some insights about the "two capacity values" phenomenon. The appearance of reaching "capacity" (indicated by high values of occupancy) in the AM peak is due to QOD occupancy expansion. However, since there are unused green times during the cycle, the real capacity has not been reached. Additionally, this phenomenon indicates that during AM peak, QOD happens more frequently and creates occupancy fluctuations. But for PM peak, although the range of occupancy values varies (changes from 0-0.8 to 0-0.4 as indicated in Fig. 8b), not all the points shift back to the left hand side (i.e. the uncongested area). There are several data points (points A-I) still staying at the congested area. Note that points $\mathrm{A}^{\prime}-\mathrm{I}^{\prime}$ in Fig. 9b represent points A-I after QOD removal.

Further investigation reveals that points A-I represent spill-over cycles, i.e. discharging flow at the intersection is blocked by downstream traffic for a period of time during the cycles. Let's take a close look at a representative spill-over cycle, Point B in Fig. 9b. The vehicle trajectories within this cycle are derived as presented in Fig. 10. As indicated in the figure, a spill-over happens at downstream leading to a blockage at the upstream intersection. A Type II QOD occurs at the advance detector at the upstream intersection, as indicated by the extended detector occupancy time during green phase. Although the QOD removal reduces the occupancy expansion caused by red interval, spill-over prolongs the detector occupation time and keeps this point stay at the high occupancy area in the AFD. This investigation also verifies that our procedure only removes the impact of Type I QOD, but not Type II.

According to the above argument, the AFD after removing QOD can be used to determine the traffic states on an arterial link. Some data points in the raw AFD staying in the congested regime with high occupancy values may not necessarily indicate that the signal link is saturated or over-saturated. It may only mean local congestion in the proximity of the detector, but no occurrence of downstream queue spillback. Only the right hand side of the revised diagrams, in which the QOD impact has been removed, adequately represents the congested situation that is caused by the blockage on downstream link. This implies that the revised AFD with QOD removal potentially offers an important approach to identify over-saturated signalized intersections (for more details, see Wu et al., 2010; Geroliminis and Skabardonis, 2010). More importantly, as we will

(a) AM Flow-occupancy relation comparison - Before and after QOD removal Flow (Veh/hour)

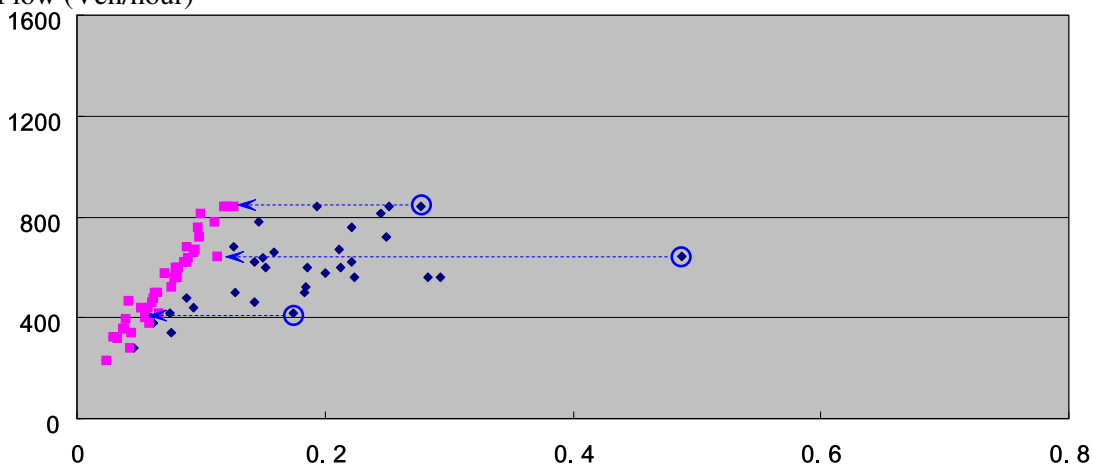

(b) PM Flow-occupancy relation comparison - Before and after QOD removal

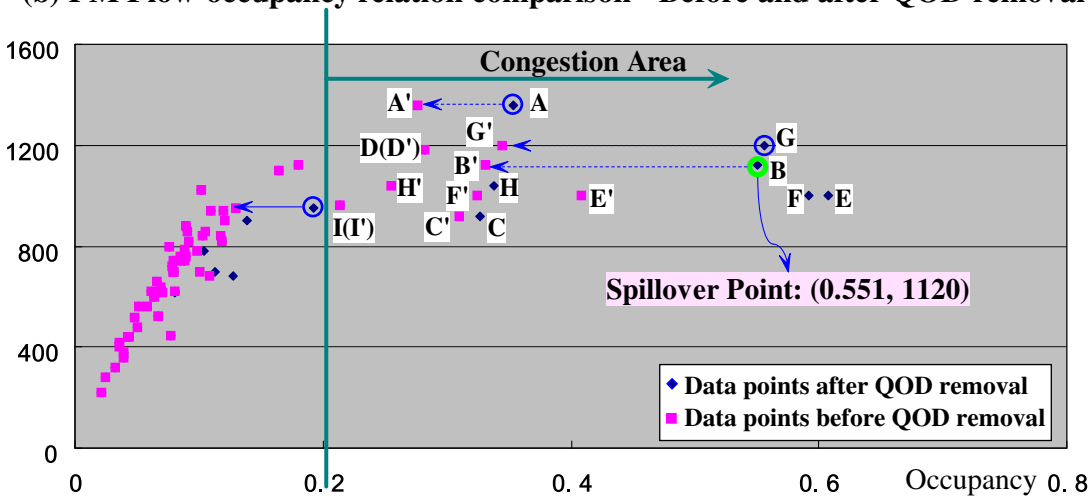

Fig. 9. AFD before and after the QOD removal (advanced detector) (a) AM peak and (b) PM peak. 
Vehicle trajectories for a cycle with spillover (Flow - Occupancy: 1120, 0.551)

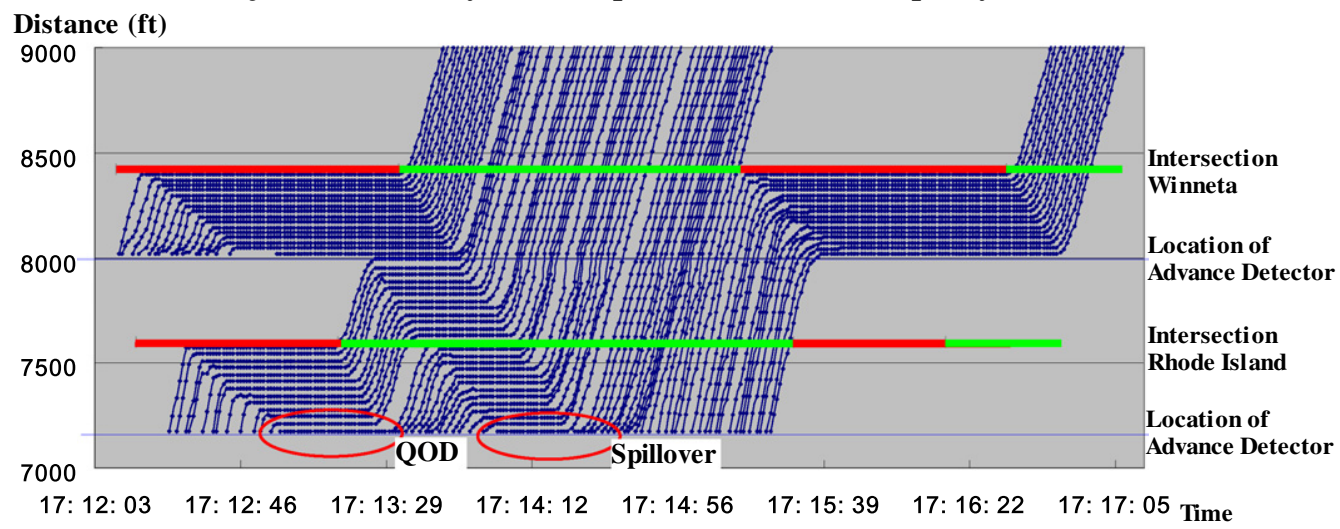

Fig. 10. Vehicle trajectories for a cycle with spill-over (point B in Fig. 9b).

further discuss in Section 6, QOD removal actually unveils a stable form of the AFD, which clearly distinguishes under-saturated and over-saturated regimes on a signal link. It should be noticed that although QOD removal successfully separates under-saturation from over-saturated conditions, it does not explain the capacity variations. As we presented in Section 4, this is explained with the effect of signal operations including $\mathrm{g} / \mathrm{C}$ ratio, turnings, and bandwidth.

\section{A stable form of the AFD}

As discussed above, QOD removal unveils an orderly and stable form of the AFD. The left hand side of the AFD indicates under-saturated traffic and the right-hand-side implies over-saturation, which specifically means spill-over from downstream intersections. Such an AFD is also observed by using 2-week's historical data collected from Rhode Island intersection (Fig. 11). Note that we do not intend to apply any statistical method to find the best fitted curve of the data in this research. But the appearance of the diagram seems to suggest a trapezoidal form, which is consistent with traditional understanding of the AFD. However, due to limited data source in congested regimes, we are not able to clearly identify the shape of the right hand side in the AFD. This deserves more effort and is left as the future research.

(a) FD - Raw Data (Advance detector, 2 weeks)

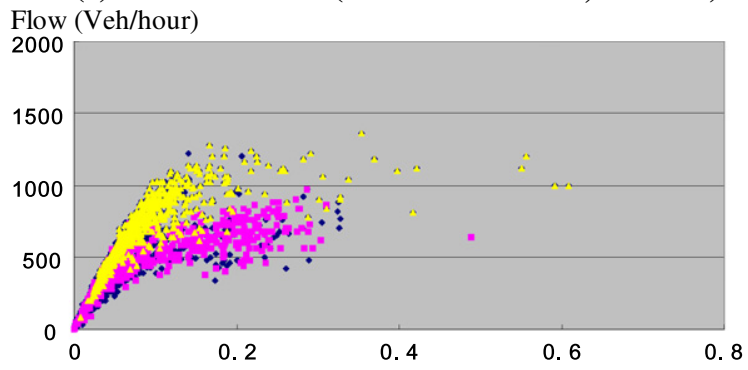

(c) FD - Remove QOD (Advance detector, 2 weeks)

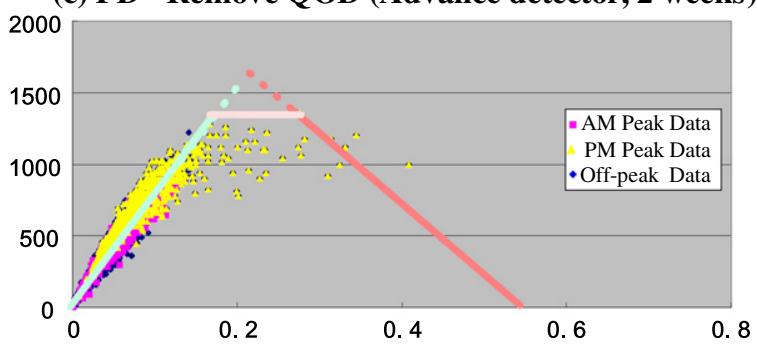

(b) FD - Raw Data (Stop-bar detector, 2 weeks)

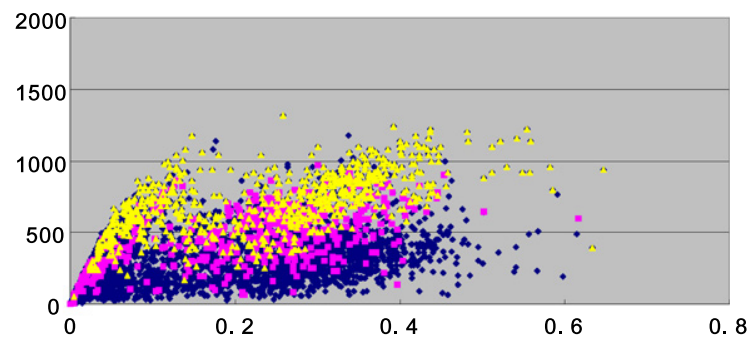

(d) FD - Remove QOD (Stop-bar detector, 2 weeks)

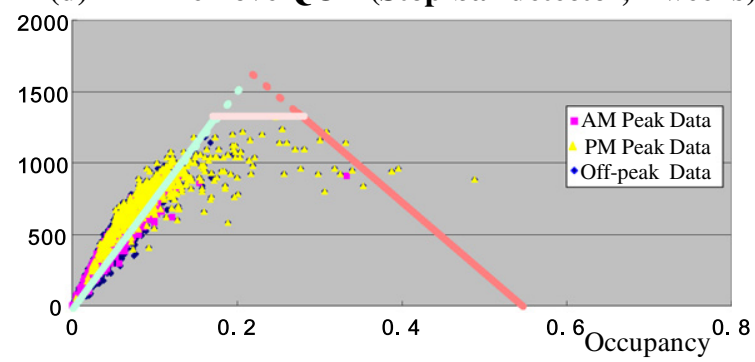

Fig. 11. The stable AFD based on 2-week's data. 


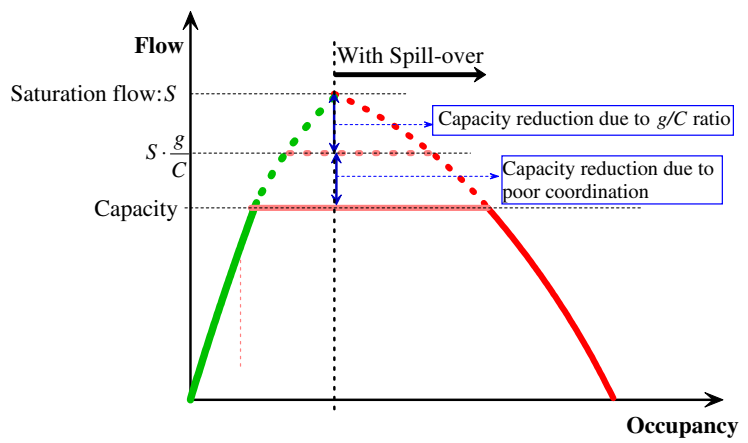

Fig. 12. Intersection capacity.

Another phenomenon demonstrated in Fig. 11 is that the data collected from advance and stop-bar detectors generate an almost identical form of the AFD (after removing QOD). This is very important because, as traffic in a signalized arterial link is inhomogeneous, the form of the AFD usually depends on where the data is collected, and how the data is aggregated. That is why the raw AFDs generated by advance and stop-bar detectors are significantly different (Fig. 11a and b). But by applying QOD removal, we uncover a stable form for the AFD. This diagram essentially represents the traffic characteristic of an arterial link, rather than that of the close-proximity of the detector location. In other words, the data collected from different detectors (stop-bar, advance or entrance/upstream) generate the similar AFDs after removal of QOD impact.

To quantify the impact of signal operations, we assume that a stable trapezoidal form of the AFD has been identified after QOD removal as shown in Fig. 12. The AFD consists of an under-saturated regime on the left hand side (green color) representing cycles without spill-over, a saturated regime in the middle (pink color), and an over-saturated regime (red color) on the right hand side. The capacity, as indicated in the figure, is restricted not only by $\mathrm{g} / \mathrm{C}$ ratio, but also by bandwidth, given the fixed turning volumes from the upstream minor approaches. The capacity value can be expressed as the minimum between the physical capacity (considering only $\mathrm{g} / \mathrm{C}$ constraint) and the possible maximum arriving flow from upstream intersection, which includes maximum volumes of upstream through movement (which is constrained by bandwidth) and the turning volume coming from upstream minor streets (see Eq. (3)).

$$
q_{i, n}^{\max }=\min \left\{\frac{g_{i, n}}{C_{i, n}} * S_{i, n}, \quad q_{i, n-1}^{A}+\frac{B_{i, n-1}}{C_{i, n-1}} * S_{i, n-1}\right\},
$$

where $q_{i, n}^{\max }$ is the capacity within $i$ th cycle for $n$th intersection; $q_{i, n-1}^{A}$ is the left and/or right turnings from the minor streets of upstream $(n-1)$ th; intersection and $g_{i, n}, C_{i, n}, B_{i, n}$, and $S_{i, n}$ are effective green time, cycle length, bandwidth, and saturation flow rate within the $i$ th cycle at the $n$th intersection. Note that the saturation flow rate may be constrained by the traffic condition at the downstream $(n+1)$ th intersection when the $n$th intersection is blocked by downstream spill-over, as described in Eq. (4):

$$
S_{i, n}=\min \left\{S_{n}^{T}, q_{i, n+1}^{\max }\right\},
$$

where $S_{n}^{T}$ is the theoretical saturation flow rate at the $n$th intersection without downstream blockage. This also partly explains that why there are vertical dispersions in the saturation regime in Fig. 11 (the other reasons could be the variable $\mathrm{g} / \mathrm{C}$ ratios caused by actuated signal control, and the inhomogeneous traffic behaviors of congested traffic).

\section{Conclusions}

This paper explores the cycle-based AFD by utilizing high-resolution event-based signal data. We demonstrate that the concentrated cloud in the arterial flow-occupancy diagram cannot be treated simply as meaningless random fluctuations; instead, it has the inner physical meaning and contains important information of traffic behavior at a signalized intersection. This paper first explains the potential reasons for the large scatter in the diagrams and it shows that the capacity is not only due to different $\mathrm{g} / \mathrm{C}$ ratios but also affected by signal coordination and turning movements. It is the combination of these two factors leading to the "two capacity values" phenomenon for AM and PM peaks.

This paper also indicated that Queue-Over-Detector (QOD) is a critical factor which shifts data points (with low flow rate) originally located in uncongested regime to congested regime, i.e. occupancy expansion. Higher frequency of QOD is also a potential indicator of poor coordination, since effective coordination reduces the chance of queue build-up in red phase. Therefore by looking at the cycle-based flow-occupancy pair, this study provides a new measure for monitoring traffic signal performance.

We also demonstrate that the flow-occupancy diagram after removing QOD impact is stable and independent of detector locations. The stable flow-occupancy diagram clearly indicates whether an arterial link is under-saturated or over-saturated with spill-over. This diagram represents the traffic characteristics of an arterial link and can be served as the base for further 
investigation of the arterial fundamental diagram and is expected to contribute to the development of the arterial traffic flow theory.

\section{Acknowledgements}

The authors would like to thank two anonymous reviewers for their insightful and constructive comments.

\section{References}

Chowdhury, D., Santen, L., Schadschneider, A., 2000. Statistical physics of vehicular traffic and some related systems. Physics Reports 329 (4), 199-329. Daganzo, C.F., Geroliminis, N., 2008. An analytical approximation of the macroscopic fundamental diagram. Transportation Research Part B 42 (9), $771-781$. Edie, L.C., 1961. Car following and steady-state theory for non-congested traffic. Operations Research 9 (1), 66-76.

Gartner, N., Wagner, P., 2004. Analysis of traffic flow characteristics on signalized arterials. Transportation Research Record 1883, 94-100.

Geroliminis, N., Daganzo, C.F., 2008. Existence of urban-scale macroscopic fundamental diagrams: some experimental findings. Transportation Research Part B 42 (9), 759-770.

Geroliminis, N., Skabardonis, A., 2010. Queue spillovers in city street networks with signal-controlled Intersections. In: Proceedings of the 89th Annual Meeting of the Transportation Research Board, Washington, DC.

Greenberg, H., 1959. An analysis of traffic flow. Operations Research 7, 78-85.

Greenshields, B.D., 1935. A study of traffic capacity. Highway Research Board Proceedings 14, 448-477.

Hall, F.L., Allen, B.L., Gunter, M.A., 1986. Empirical analysis of freeway flow-density relationships. Transportation Research Part A 20 (3), 197-210.

Hallenbeck, M.E., Ishimaru, J.M., Davis, K.D., Kang, J.M., 2008. Arterial performance monitoring using stop bar sensor data. In: Proceedings of the 87th Annual Meeting of the Transportation Research Board, Washington, DC.

Helbing, D., 2009. Derivation of a fundamental diagram for urban traffic flow. European Physical Journal B 70 (2), $229-241$.

Kerner, B.S., 2004. Three-phase traffic theory and highway capacity. Physica A: Statistical Mechanics and its Applications 333, 379-440.

Koshi, M., Iwasaki, M., Okhura, I., 1983. Some findings and an overview on vehicular flow characteristics. In: Proceedings of the 8th International Symposium of Transportation and Traffic Theory, Toronto, Canada, pp. 403-426.

Lighthill, M.J., Whitham, G.B., 1955. On kinematic waves: II. A theory of traffic flow on long crowded road. Proceedings of the Royal Society A 229 (1178), 317-345.

Liu, H., Ma, W., 2009. A virtual probe approach for time-dependent arterial travel time estimation. Transportation Research Part C 17 (1), 11-26.

Liu, H., Wu, X., Ma, W., Hu, H., 2009. Real-time queue length estimation for congested signalized intersections. Transportation Research Part C 17 (4), $412-$ 427.

Newell, G.F., 2001. A simplified car-following theory: a lower order model. Transportation Research Part B 36 (3), $195-205$.

Wu, X., Liu, H., Getttman, D., 2010. Identification of oversaturated intersections using high-resolution traffic signal data. Transportation Research Part C 18 (4), 626-638.

Underwood, R.T., 1961. Speed, volume, and density relationships: quality and theory of traffic flow. Yale Bureau of Highway Traffic, $141-188$. 